\title{
Biotecnología: Nuevos paradigmas y ruptura de pactos
}

Biotechnology: New paradigms and breaking agreements

Profesor Serge Boarini ${ }^{1}$.

\author{
https://doi.org/10.5377/rcfh.v5i2.8911
}

${ }^{1}$ Profesor Asociado, Doctor en Letras-Filosofía. Colegio Superior del Oiselet, Miembro del Consejo Superior de Biotecnología, Francia

Correspondencia a: serge.boarini@ac-grenoble.fr

\section{CITAR COMO}

Boarini S. Biotegnología: Nuevos paradigmas y ruptura de pactos. Rev. cienc. forenses Honduras. 2019; 5(2): 36-46

\section{ASPECTOS BIOETICOS}

El autor declara que no hubo conflicto de interés en la preparación de este artículo. Todas las opiniones aquí vertidas son responsabilidad exclusiva del autor.

Este texto es una versión abreviada de una declaración escrita para el Foro Bioético y Desarrollo Sostenible Franco-Latinoamericano, celebrado en San José, Costa Rica del 2 al 3 de mayo de 2019.

\section{AGRADECIMIENTOS:}

Agradezco a Christian Byk y Alfonso Chacón Mata, los organizadores, por su inagotable generosidad, y agradezco a Mireya Matamoros Zelaya por su gran confianza.

Este trabajo fue escrito en francés, se agradece a Valeria Alonzo Matamoros y a Keyla Marcia, por su colaboración en la traducción.

\section{RESUMEN}

La biotecnología introduce una relación sin precedentes con la naturaleza: rompe con el paradigma de las técnicas convencionales al introducir una nueva temporalidad, al emancipar los modos de producción de la tutela humana, al al evitar cualquier revisión de las formas producidas y cualquier retorno a un estado anterior. De ello se deduce que la biotecnología rompe tres pactos, que permanecieron tácitos hasta su advenimiento, lo que nos une a los hombres, a la naturaleza, a las generaciones futuras: pacto de equidad-solidaridad, pacto de reciprocidad-confianza, pacto de responsabilidad solidaridad. Finalmente, se describen algunas pistas para contener las consecuencias de estas infracciones de pactos, y tal vez, para restablecer el equilibrio perdido por un cierto uso de la biotecnología.

\section{PALABRAS CLAVE}

Bioética, Biotecnología, Generaciones futuras.

\section{ABSTRACT}

Biotechnology introduces an unprecedented relationship with nature: it breaks the paradigm of conventional techniques by introducing a new temporality, by emancipating the modes of production of human guardianship, by avoiding any revision of the forms produced and any return to a state previous. It follows that biotechnology breaks three pacts, which remained tacit until its advent, which unites us to men, to nature, to future generations: equity-solidarity pact, reciprocity-trust pact, responsibility pact solidarity. Finally, some clues are described to 
contain the consequences of these covenant violations, and perhaps, to restore the balance lost by a certain use of biotechnology.

\section{KEYWORDS}

Bioethical, Biotechnology, Future generation.

\section{INTRODUCCIÓN}

Supongamos que recibo de las manos de mi padre el libro escolar de su padre (mi abuelo). Lo coloco frente a mí, obviamente en medio de los libros de trabajo de mi biblioteca. Así, un objeto fue transferido de su propietario a otra persona que lo sucedió en el orden familiar sin que se codificara esta transferencia (el libro no aparece en los bienes patrimoniales establecidos a la muerte de mi abuelo), sin que esta transferencia fuese premeditada (mi abuelo murió sin conocer el embarazo de mi madre), sin que esta transferencia fuera ordenada por un deber (mi padre podría haber optado por regalar este libro o destruirlo sin que nada, ni nadie pudiese lamentarlo). Este libro fue transmitido de un ausente a otro ausente, sin un contrato entre ambos (uno desaparecido, el otro aún no llegó), sin crear compromiso. Sin embargo, lo cuido y me hubiera enojado si este libro hubiera sido vendido, degradado, destruido, aunque no puedo acreditar ningún derecho a la integridad de este último.

¿Se puede transponer este ejemplo y arrojar luz sobre el "legado" que haremos a las generaciones venideras? Si el mundo ocupado por uno de ellos se transmite a otros de tal manera que este último pueda encontrar plena satisfacción, mientras que ninguna de las partes tendrá conocimiento personal y nominativo o general de los demás, con la mera presunción de que otros vendrán después de nosotros ¿Cuál es la naturaleza de este deber de transmitir un mundo en el que una vida humana y una vida deseable, es decir, una vida tal que las aspiraciones de los hombres puedan encontrar plena satisfacción, aún sea posible?
Estos problemas solo pueden abordarse después de considerar qué la biotecnología está impulsando nuevas relaciones en el mundo. En esta primera parte de un estudio más completo; me gustaría establecer que la biotecnología rompe con el paradigma de las tecnologías conocidas antes que ella (I), luego mostraré cómo la biotecnología rompe con tres pactos, tácitos hasta su advenimiento; como nos vinculamos con los hombres, con la naturaleza y con las generaciones futuras (II).

\section{Tecnología y biotecnología. ¿Romper el paradigma?}

La biotecnología tiene características específicas que la distinguen de las tecnologías pasadas: ya no se encuentran en la misma temporalidad; produce artefactos autónomos; cualquier reversibilidad y cualquier capacidad de revisión son imposibles.

Temporalidad. En la tecnología como en la biotecnología, las temporalidades y modalidades de la acción humana son diferentes. La temporalidad de la tecnología es corta: el resultado de la acción tecnológica debe ser lo más cercano posible a lo que la ha permitido debido a varios factores: el financiamiento, el despliegue de recursos humanos y técnicos, la organización y planificación implementada para lograr estos resultados. Primero por razones financieras, la inversión debe ser amortizada, también por razones de perspectiva y esperanza, las consecuencias son el tema de fuertes expectativas. Pero nuevamente por razones relacionadas con la naturaleza de la tecnología: es necesariamente a corto plazo. El tiempo de la operatividad parece el tiempo de prorroga; la técnica funciona como magia: la instantaneidad es soñada, deseada, realizada si es posible. Las células fotoeléctricas reemplazan y satisfacen el deseo de las fórmulas de cuentos "ábrete Sésamo" 
cuando las puertas móviles se abren al paso. Las tecnologías toman poco tiempo mientras que la biotecnología actúa durante mucho tiempo, trayendo consecuencias y efectos indirectos. Ya sea que haya difusión (consecuencia) o empoderamiento de nuevas formas de vida o el empoderamiento de ecosistemas sin precedentes (efectos indirectos), la biotecnología rompe con el paradigma de las tecnologías industriales. Es similar a la tecnología nuclear para la cual el largo tiempo es de suma importancia, (reciclaje de residuos, entierro, desmantelamiento de instalaciones, pero también contaminación de ambientes irradiados, cuidado de poblaciones contaminadas o que viven en nuevas condiciones) Empoderamiento.

Heidegger M. ${ }^{1}$ distingue, por un lado, la tecnología que acompaña y realiza el proceso de la naturaleza, y por otro, la técnica moderna que provoca la fuerza de la naturaleza, la libera y la aleja de sus leyes ordinarias ${ }^{1}$. Debe agregarse a esta distinción y reservar un nuevo lugar para la biotecnología. La realización de todas las cosas posibles soñadas por Bacon, se hace efectiva: las levaduras producen opioides ${ }^{2,3}$.

Con la biotecnología, hay al mismo tiempo acompañamiento (Los organismos genéticamente modificados (OGM) siguen las leyes del desarrollo de la naturaleza), provocación (introducción de una nueva secuencia de ADN) pero también penetración en los principios de organización de la vida, potenciación de la vida así creada, alteración de ecosistemas inclusivos. El empoderamiento significa un abandono de organismos nuevos en la naturaleza mediante la polinización, por ejemplo; los trastornos del ecosistema afectan el ecosistema natural, pero también impacta el ecosistema del cual el hombre es parte (ecosistema inclusivo): modifica el comportamiento humano en su propia naturaleza.
El aumento de pesticidas y herbicidas para tratar cultivos genéticamente modificados está afectando la salud humana ${ }^{4}$. Más confinado, los riesgos de diseminación de los transgenes utilizados en la terapia génica que deben estar contenidos en dispositivos de seguridad (guantes, limpieza de jeringas, máscaras).

\section{Reversibilidad y revisibilidad imposibles.}

La modalidad de la temporalidad de la acción humana para las tecnologías previas a la explotación de los recursos fósiles es la reversibilidad y la revisibilidad.

La reversibilidad se refiere a la propiedad de cancelar el curso temporal de un proceso: el tiempo de este tipo de tecnología es el tiempo de la única cronología, no se producen cambios sustanciales para evitar el cuasi retorno ${ }^{5}$.

La revisibilidad se refiere a la propiedad del reajuste cuantitativo o la reapropiación cualitativa de un proceso: revisión de los pronósticos de producción (reducción de las cantidades a producir, adaptación del reclutamiento y asignación de personal); revisibilidad de los procesos de producción (uso de sustitutos en caso de escasez - remolacha contra caña de azúcar, con diferentes modos de refinación).

Un proceso tecnológico puede ser reversible sin ser revisable: el uso de norias y molinos que utilizan fuentes de energía capturadas sin ser desviadas en su naturaleza (no sabíamos cómo

\footnotetext{
1 "Ella" [la técnica en Aristóteles] revela lo que no le sucede a uno mismo y aún no está ante nosotros, lo que puede tomar, a veces esta apariencia, este turno, a veces otra. Quien construye una casa o un bote, quien da forma a una copa de sacrificio revela lo que se producirá de acuerdo con las perspectivas de las cuatro modalidades del "por venir", Heidegger M.

1."La presentación que gobierna la tecnología moderna es una provocación [Herausfordern] por la cual la naturaleza está llamada a entregar energía que como tal puede ser extraída [Herausgefordert] y acumulada" Heidegger M.

2."Nuestra Fundación tiene el objetivo de[..] hacer retroceder los límites del imperio humano para lograr todo lo posible", Bacon.
} 
hacerlo de otra manera); un proceso tecnológico puede ser revisable sin ser reversible: el uso de compuertas para producir electricidad puede sufrir modificaciones (tamaño de las tuberías, materiales de las tuberías, transformación de la fuerza motriz) sin que sea posible el retorno a un estado producción de energía, ya que la demanda ha aumentado; puede ser revisable y reversible como lo demuestran los esfuerzos de Colbert para mantener los bosques ${ }^{6}$.

Las tecnologías previas a la explotación de los recursos fósiles están al mismo tiempo en un presente perpetuo (una edad de oro) y en un retorno de este, si hay una transformación de detalles. Las tecnologías de la era de los recursos fósiles son revisables sin ser reversibles: ¿ el cese de los Cloro Fluoro Carbonos CFC (Protocolo de Montreal), ¿reparará el daño causado por su uso? El orden tecnológico se centrará en los modos de explotación, transporte, transformación y uso de estos recursos. La explotación del gas esquisto es una revisión de los modos de explotación de los recursos energéticos sin que eso cause una reversión del modo de producción. Sin duda, dos características de este proceso tecnológico son la globalización de estos recursos en el sentido de que se dibujan y transforman sin límites políticos limitados a priori y distancias geográficas (gasoductos, tuberías, líneas de alta tensión), el otro el agotamiento previsible de estos recursos y su transformación irreversible después del consumo (gases de efecto invernadero). Es la salida de la edad de oro (imagen para designar el tiempo detenido en un orden considerado estable) y la entrada en la historia (imagen para designar la constitución de un sentido en una sucesión temporal, lo que significa intencional, por ejemplo, la organización de ciudades en las que ingresan las autopistas, o significado indirecto, por ejemplo, el desarrollo de servicios de salud para luchar contra las alergias debido a las fuentes de contaminación). Las tecnologías que son más antiguas que la era de la explotación de combustibles fósiles, incluida la energía nuclear y la biotecnología, son representativas y comparten la irreversibilidad con las anteriores. Así, los organismos creados son, por esta razón quienes disfrutan de nuevas propiedades. Pero estas tecnologías agregan a las anteriores la falta de capacidad de revisión. No habría medidas correctivas para los posibles riesgos y los riesgos reales. Tanto la radiactividad como la propagación del polen de las plantas transgénicas no se pueden retener en el espacio, ni contener en el tiempo, ni reparar.

\section{Obras "Producidas"}

La especificidad de la biotecnología es entrar en la vida para que se convierta en un trabajo en progreso. La biotecnología delega a los vivos y crea una cierta capacidad de acción (que supone limitada y contenida - Tecnologías Terminator de semillas Monsanto) para intervenir por sí misma en el medio ambiente de la vida. Los temores sobre la nueva tecnología Gene Drive se alimentan de esta nueva potencia. Así, lo que fue trabajo humano se emancipa de la tutela y funciona a su vez mediante la movilización de agentes externos (las abejas para plantas genéticamente modificadas (PGM)). Es aquí donde se plantea la cuestión de los pactos y la ruptura de estos.

\section{II. ¿Rompimientos de pactos?}

Más allá de los momentos reales de crisis, la cuestión sigue siendo la realidad de la ruptura de

\footnotetext{
5.- "La inflación, una sequía catastrófica o una caída del mercado de valores no deja absolutamente ningún rastro en la economía. La reversibilidad completa es la regla general, al igual que en la mecánica.

6.-Intervenir por sí mismo en el entorno de la vida. Los temores sobre la nueva tecnología Gene Drive se alimentan de esta nueva potencia. Así, lo que fue trabajo humano se emancipa de la tutela y funciona a su vez mediante la movilización de agentes externos (abejas para PGM). Es aquí donde se plantea la cuestión de los pactos y la ruptura de estos diferentes pactos.
} 
los pactos de equidad-solidaridad, reciprocidadconfianza y solidaridad-responsabilidad, por parte de la biotecnología. ¿Existen tales pactos? ¿Las biotecnologías están rompiendo con estos pactos?

\section{Pacto de equidad-solidaridad.}

La biotecnología interrumpe los vínculos existentes entre los seres humanos, independientemente de sus agrupaciones (política en las sociedades, comercial en las unidades de producción, distribución social en el territorio).

Por un lado, la investigación y la innovación solo pueden apoyarse con la ayuda de importantes medios financieros, cuya amortización solo se puede encontrar mediante la transferencia de derechos por encima de la capacidad de pago de aquellos que podrían beneficiarse de estos descubrimientos. Al plantearse como alternativas a la ausencia de bienes primarios, las biotecnologías crean una expectativa que no puede satisfacerse para las poblaciones que más lo necesitan. De ahí el círculo de pobreza: la ausencia de bienes primarios haría necesario el acceso a la biotecnología para compensar las deficiencias de la producción natural; el uso de estas biotecnologías requiere medios financieros que la ausencia de recursos impide obtener; poseer los derechos para usar estas biotecnologías requiere medios pecuniarios.

Por otro lado, para el ejercicio del pacto de equidad-solidaridad, la existencia de un compromiso entre todos los residentes de una comuna, entre todos los ciudadanos de un Estado, entre los propios Estados, nada obvio. Los detalles de la modalidad de tal compromiso son variables y proteicos dependiendo de la naturaleza del vínculo social: socios económicos, empleados de una empresa productora o un subcontratista o una empresa competidora, residentes locales, residentes de un mismo municipio, de la misma región ... y con la complejidad de estas diversas afiliaciones (por ejemplo, el residente local puede trabajar en otro territorio). Finalmente, los grupos humanos que se ponen en contacto ya no son administrados principalmente por reglas de naturaleza política. Los límites que podrían imponer los estados se enfrentan a la ausencia de legislación internacional que no sea, por ejemplo, los acuerdos de la Organización Mundial del Comercio (OMC).

Algunas respuestas son posibles. Por lo tanto, la desterritorialización de las empresas que poseen patentes y la liberalización del comercio están causando una distribución de riqueza y habilidades. Si se producen flores transgénicas de color violeta-púrpura, cortadas en América del Sur, llegan a los mercados de Australia, EE. UU y Japón. Además, la disparidad en la asignación de conocimientos y la distribución de bienes no es específica de la biotecnología. Pero con respecto a esto último, la disparidad puede alcanzar las mismas condiciones de la vida humana al evitar la producción tecno-agrícola donde se esperaría, y al no permitir que las poblaciones accedan a nuevos servicios de salud (terapias genéticas).

\section{Incumplimiento del pacto de reciprocidad- confianza}

El pacto de reciprocidad-confianza, el de la Casa Común ("El desafío urgente de salvaguardar nuestra casa común ..." "Nuestra casa arde ..." "La Tierra, hogar de la humanidad") ${ }^{7-9}$, por la cual el hombre concede la confianza y la protección en el entorno que lo rodea no parece tener sentido: el entorno no parece ser capaz de comprender, aceptar y compartir los compromisos humanos: "Es imposible entrar en un pacto con los animales, porque les falta la palabra por la cual podrían significar su voluntad"10. El texto en inglés de Hobbes es más elaborado: se adhiere a las bestias salvajes a las que no se pueden transferir los 
derechos por falta de, para estas bestias, poder comprender nuestro idioma. No pueden entender ni aceptar tal transferencia; ni pueden transferirse derechos el uno al otro ${ }^{11}$.

La biotecnología conduce directamente o por consecuencias distantes a otra relación con el mundo vivo. Por un lado, todo se transforma en cosas. De la naturaleza griega, representada en el modelo de crecimiento y respiración, un ciclo permanente y reversible, no queda nada ya que todo se ha convertido en "cosa", luego en "bueno" y luego en "mercancía" o "servicio". La cosa tiene una existencia independiente, mientras que lo bueno es apropiado ${ }^{12}$ (lo bueno es cosa de alguien). La mercancía transforma todo bien en un elemento que tiene valor para los demás, este valor se convierte o establece con mayor frecuencia en una moneda ${ }^{13}$. El servicio es una mercancía desmaterializada (una acción es el objeto de una compra) o un servicio para terceros. La intención de la biotecnología, el significado de su actividad considerada fuera de cualquier organización práctica (financiera, legal, administrativa, política) es convertir el mundo de las cosas en un mundo de beneficios: servir a las cosas en el mundo de las necesidades humanas. Tal sería el caso de la terapia génica. La realización de esta intención, la entrada de biotecnologías en un mundo que organiza la producción de servicios o la conversión de cosas en bienes, provoca la transformación de la cosa en mercancía: los elementos naturales son apropiados (la berenjena india de Monsanto) ${ }^{14}$. Al hacerse cargo de los análisis y los términos de N. Georgescu-Roegen, la biotecnología sería, en el orden del tiempo, el último invento en tomar baja entropía (cuya energía es útil y gratuita) en el stock de tierra para liberar alta entropía en forma de residuos (cuya energía es inutilizable y atada). Lo ideal sería producir más energía de la que se consume o, si el balance es cero, asegurar que la energía inutilizable no haga imposible consumir el stock de tierra restante.

Por otro lado, no toda la biotecnología es revisable y no toda es reversible. La entrada en las propiedades de la materia viva puede desencadenar procesos autónomos. Cualquier técnica, antes de la era industrial, está en la modalidad de dependencia, mantenimiento y monitoreo. Los efectos tecnológicos dependían del conocimiento humano y cesaron con este conocimiento, no sin matices ya que, por ejemplo, la civilización sumeria se habría derrumbado debido a que lo que hizo que esta civilización fuera, lo que fue, fue la fuerza del riego ${ }^{15}$. Pero cuando la tecnología se liberó del poder y el control humano, provino de otros factores (falta de perspectiva o perspectiva sobre la tecnología utilizada, búsqueda desenfrenada de ganancias, sobreexplotación de recursos). Con la era industrial, los materiales se están liberando a sí mismos y a ciclos cortos de la naturaleza y el poder humano: los desechos ya no son comparables a la escala generacional humana. Con el telar manual, el artesano impulsa su ritmo a la máquina; La automatización hace que el trabajador vaya al ritmo permitido por la nueva tecnología y deseado por los organizadores del trabajo ${ }^{16}$.

\footnotetext{
12.Los "bienes" son objetos físicos producidos para los cuales existe una demanda, sobre los cuales se pueden establecer derechos de propiedad y cuya propiedad se puede transferir de una unidad institucional a otra a través de una transacción de mercado ( 13."Bienes" significa bienes que aumentan o reducen el stock de recursos materiales de un país o territorio al ingresar (importar) en el territorio económico del país o al abandonarlo (exportaciones) ", Organización Mundial del Comercio. International Trade Statistics 2011. "A los efectos de IMTS 2010 y con referencia al SCN 2008, los bienes se definen como objetos físicos producidos para los cuales existe una solicitud, sobre los cuales se pueden establecer derechos de propiedad y cuya propiedad puede ser transferido de una unidad institucional a otra a través de transacciones de mercado, así como ciertos tipos de productos basados en la captura de conocimiento almacenado en medios físicos que pueden cruzar las fronteras físicamente ".
} 
Las tecnologías preindustriales están en la escala generacional humana. El temor a la biotecnología es el de una liberación desordenada e incontrolada de los efectos de estas tecnologías, así como un empoderamiento tanto de los efectos como de los procesos creados. La resistencia de las malas hierbas, como las que se vuelven resistentes al glifosato (plantas Roundup Ready), la adaptación de las plagas a insectos y la alteración de los ecosistemas (la mosca del olivo en España, los mosquitos de Brasil con un nuevo gen, dependiente de un antibiótico, la tetraciclina) son algunos ejemplos.

\section{Ruptura del pacto responsabilidad-solidaridad}

La biotecnología coloca al ser humano ante una responsabilidad sin precedentes e imprevista frente a las generaciones futuras. Lo que plantea dos paradojas.

La primera paradoja es responder a las generaciones que aún no han nacido sin ninguna posibilidad de reparación. El artículo 1240 del Código Civil francés estipula: "Cualquier hecho de cualquier hombre, que cause daño a otro, lo obliga a repararlo". Pero ¿el código civil, puede "aplicarse a" las sociedades? y aún más, ¿ puede ser aplicable para generaciones cuya delineación es una tarea delicada? Entonces, ¿a quién atribuir la responsabilidad si ningún criterio permite discernir cuándo comienza o cuándo se detiene una generación? También es necesario que sea posible atribuir la responsabilidad de las decisiones de una generación anterior que haya puesto en peligro una nueva generación. ¿Las consecuencias para la salud pública del uso de productos fitosanitarios se derivan de su primer uso, o resultan del incum plimiento de las primeras regulaciones? ¿Cómo podrían hacer daño aquellos que ya no están (consecuencias distantes, imprevistas e impredecibles de sus decisiones), y aún más cómo podrían sufrirlo aquellos que aún no están? Más aún, aquellos que no son, surgirán solo por el hecho de nuestra voluntad (o nuestra negligencia, lo que sería otra dificultad: ¿aquellos que ven el día sin nuestro conocimiento podrían reclamar el pleno disfrutar de sus derechos y exigir respeto por ellos?; Nos preguntamos, ¿Podrían las generaciones futuras invocar prejuicios no relacionados con su nacimiento, sino con las circunstancias bajo las cuales este nacimiento los creó?, ¿un mundo sin agua, sin tierra cultivable, por ejemplo?

Una segunda paradoja sería la responsabilidad de las cosas comunes (mar, atmósfera, pero también animales salvajes para la ley romana ${ }^{17}$ ) a menos que las haya dispuesto entre las cosas apropiadas (versión fuerte), o a menos que las guarde, entre el uso de las actividades privadas (versión débil). Pero al hacerlo, reconocemos el derecho de la generación anterior a poseerlo y en consecuencia, a poder transformarlos como lo desee. Especialmente debido a que la distinción podría llevarse más allá: el mar sería algo común, el agua que se extrae podría ser buena, pero luego las partículas de agua y sus propiedades o incluso los elementos vivos y no vivos, quienes viven allí por accidente, ¿'son comunes o apropiados?

El pacto responsabilidad-solidaridad se trata más de confianza y promesa. Ninguna generación puede aparecer ante la que le seguirá; la que sigue no podrá abordar la que le precedió, más que por protestas como las generaciones jóvenes alemanas con respecto a que sus mayores hayan conocido el nazismo.

Sin embargo, postulado así, los términos del problema están sesgados: ninguna generación da

16. “El tejedor en su mano teje rápida o lentamente mientras respira lenta o rápidamente; él actúa como vive; el oficio se ajusta al hombre. Allí, por el contrario, es necesario que el hombre se ajuste a la profesión, que el ser de sangre y carne donde la vida varía según las horas sufra la invariabilidad de ser de acero". 
inmediatamente el paso y el lugar a la siguiente. Las generaciones coexisten; uno educa y prepara al otro. Como resultado, se pueden asignar responsabilidades, por ejemplo, criminalizando ciertas decisiones por sus consecuencias, como los crímenes de lesa humanidad. La generación anterior puede organizar la toma de decisiones haciéndolas públicas, transparentes, leales, precisas y completas.

Una de las principales preocupaciones, en el caso de que se elija el entierro de residuos radiactivos, es perpetuar la memoria de los lugares de depósito. Se ofrecen varias opciones: confianza en la auto organización de las empresas privadas y el juego libre del mercado; autorregulación de la vida pública permitiendo que se expresen los intereses divergentes; la creación y promoción de cuerpos deliberativos, cámaras de eco de voces divergentes; instituir un "Pugwash" de la biotecnología ${ }^{18}$, un organismo independiente de expertos; delegación de habilidades a la política internacional para la toma de decisiones.

\section{PARA CONCLUIR}

La biotecnología ha sido concebida como uno de los medios para garantizar el desarrollo sostenible al permitir que la producción de alimentos esté en línea con las crecientes necesidades del planeta, al limitar los impactos ambientales de esta producción, al hacer posible la explotación. Fuentes de energía nuevas y renovables. Los temores que alimentan son los de romper el pacto de equidad al poner el conocimiento y la tecnología en manos de algunos e imponer a otros la carga de comprarlos o recompensar a los titulares de patentes ${ }^{19}$, el de romper pacto de reciprocidad-confianza al socavar un orden natural dado como inmutable en el peor de los casos, tan autónomo al menos, como garante de
La biotecnología está impulsando nuevas relaciones en el mundo y. rompe con el paradigma de las tecnologías conocidas antes que ella.

Pero además rompe con tres pactos, tácitos hasta su advenimiento; como nos vinculamos con los hombres, con la naturaleza y con las generaciones futuras

\section{Coloca al ser humano ante una responsabilidad sin precedentes e imprevista frente a las generaciones futuras.}


la sostenibilidad de la vida en el mejor de los casos, el de la ruptura del pacto de responsabilidadsolidaridad al iniciar una investigación de manera que la humanidad que sobrevivirá será diferente de lo que es. Las preguntas clásicas sobre el derecho a la acción concertada con otros se vuelven a plantear en aras de compartir los beneficios, el derecho a la acción en el mundo con la preocupación de la gestión del buen padre que no agotará los recursos, el derecho a una acción responsable y prudente con la preocupación de dejar un mundo habitable para las generaciones futuras.

Varios supuestos subyacen a la afirmación de una adaptación de la biotecnología desde la perspectiva del desarrollo sostenible.

Con respecto al pacto de equidad y solidaridad, los medios de cambio deben suscribirse a las siguientes condiciones:

1.-Los elementos constitutivos de la identidad de los seres vivos son cosas que están fuera del comercio, incluso "cosas comunes"; el título del proceso no debe privar a una parte de la población de los beneficios que puede esperar (limitación de la duración de estas patentes a lo largo del tiempo, reducción preferencial de los precios de comercialización, asignación inmediata de derechos de bienes en caso de peligro de parte de la población).

2.-El conocimiento adquirido y las técnicas elaboradas no pueden ser argumentos para oponerse a su transmisión (acceso a este conocimiento para los Estados en situación de peligro sanitario). La apropiación del conocimiento biotecnológico seguirá siendo una derogación del principio de una universalidad imprescriptible de un patrimonio común del conocimiento.
En vista del pacto de reciprocidad-confianza, los cambios producidos por estas tecnologías deben ser tales que puedan ser reversibles, revisables, compensables: se debe prever la compensación. Con respecto al pacto responsabilidad-solidaridad, los cambios deben hacerse para dar a las generaciones futuras, sin reservas, el mundo en el que la generación actual quisiera vivir, con la garantía, que con el estado del conocimiento presente, tanto como sea posible, proporcionar los medios y poder limitar los riesgos posibles en el futuro.

19."Además, los miembros del foro internacional no gubernamental ya no deberían ser responsables de las empresas de biotecnología, sino simplemente científicos eminentes, que pueden sopesar los problemas de manera apasionada". Un posible modelo para tal foro sería la Conferencia Pugwash, un cuerpo consultivo no oficial de científicos que funcionó durante la guerra contra los peligros nucleares y la composición de estos.

20."El verdadero desafío es garantizar que las nuevas tecnologías lleguen a todos los que las necesitan, superando desafíos como la falta de información y, en algunos casos, la incapacidad de pagar por las tecnologías desarrolladas en un contexto".

\section{REFERENCIAS BIBLIOGRÁFICAS}

1. Heidegger M. La question de la technique. In : Essais et conférences [Vorträgeund Aufsätze, Pfullingen, 1954]. Paris, Gallimard, 1958. p. 18-20.

2. Bacon C, Francis, La nouvelle Atlantide. Paris, Flammarion, 1995.

3.-Van Eeckhout L. Monsanto condamné pour I'intoxication d'un agriculteur français, Le Monde, 11 septembre 2015.

Disponible sur https://www.lemonde.fr/planete/article/2015/09 /11/monsanto-condamne-pour-l-intoxication-dun-agriculteur-francais 4751628 3244.html [Consulté le 27 novembre 2019]3.

4.-Galanie S, Thodey K, Trenchard Isis J, Filsinger Interrante M, Smolk C. D. Complete biosynthesis of opioids in yeast. Science, 04 Sep 2015 : Vol. 349, Issue 6252, pp. 1095-1100. DOI: 10.1126/science.aac93734. 
5. Georgescu Roegen N. La décroissance. 3 ed. Rev aug. Paris: Ellébore, Sang de la terre ; 2006.

6. Larrère $C$, Larrère $R$. Du bon usage de la nature: Pour une philosophie de l'environnement. Champs essais. Paris: Flammarion; 2009. 355 Seiten.

7. Albareda Tiana S. The Contributions of Laudato si' within the Context of the 2030 Agenda for Sustainable Development. Scripta Theologica. 2016; 48(2):443-462. doi: https://doi.org/10.15581/006.

8. Chirac J. Déclaration de M. Chirac. [Internet]Johannesburg ;2002. [consultado 4 octubre 2019]. Disponible en: https://www.viepublique.fr/discours/132014-declaration-de-m-

jacques-chirac-president-de-la-republique-sur-la$\underline{\text { mob }}$

9. Nations Unies sur I'environnement et le développement. Sommet Planete Terre. Déclaration de Rio Sur L'environnement Et Le Développement Principes de Gestion Des Forêts: Conférence des Nations Unies sur l'environnement et le développement Rio de Janeiro, Brésil 3-14 juin 1992 [Internet]. New York: Document non officiel Départment de l'information, Organisation des Nations Unies; 1993. [consultado 12 julio 2018]. Disponible en:

https://www.un.org/french/events/rio92/riofp.htm

10. Hobbes T. Léviathan. [1668, tr. latine]. Paris, Dalloz/Vrin, 2004.

11. Hobbes T. Leviathan. [1651]. London: Penguin Books ; 1988. P.168

12. Nation Unies. Statistiques du commerce international de marchandises: Concepts et définitions, 2010. [Internet]. New York: Nation Unies; 2012. Annexes, concepts el définitions de base de la comptabilité nationale et de balance des paiements ; p.83. [consultado 4 octubre 2019]. Disponible en:

https://unstats.un.org/unsd/trade/egimts/IMTS\%202010\%20(French).pdf
13. Nation Unies. Statistiques du commerce international de marchandises: concepts et définitions, 2010. [Internet]. New York: Nation Unies; 2012. Chapitre I, Portée et date d'enregistrement;p.13-14. [consultado 4 octubre 2019].Disponible en

https://unstats.un.org/unsd/trade/egimts/IMTS\%202010\%20(French).pdf

14.-J. Bouissou, Monsanto poursuivi pour "biopiraterie" par l'Inde, Le Monde, 17 août 2011. Disponible sur https://www.lemonde.fr/planete/article/2011/08/ 17/monsanto-poursuivi-pour-biopiraterie-par-Iinde 1560365 3244.html [Consulté le 27 novembre 2019]

15. Berger A, Perthuis $C$ de, Perin N. Le développement durable. Repères pratiques. Paris: Nathan; DL 2014 .

16. Jules M. Le Peuple. 5ed. Paris : Gallica; 1877. p. 38.

17. Jadot B. L'environnement n'appartient à peronne et son utilisation appartient à tous. In: Ost F, Gutwirth S, direct. Quel avenir pour le droit de l'environnement, Actes du colloque organisé par le CEDRE, Centre d'étude du droit de l'environnement - F.U.S.L. et le CIRT, Centrum interactierecht en technologie - V.U.B. Bruxelles: Facultés Universitaires Saint-Louis ; 1996. p. 95.

18. Wilkins AS. Sustainable development, biotechnology and the North-South divide. Bioessays. 2003; 25(2):97-8. doi: 10.1002/bies.10242.

19. Commission des Nation unies sur l'environnement et le développement. Notre avenir à tous : Rapport Brundtland.[Internet]. NY: CNUED ; 1987. [consultado 4 octubre 2019]Disponible sur https://www.diplomatie.gouv.fr/sites/odysseedeveloppementdurable/files/5/rapport_brundtland.pdf 